\title{
Tumor de Wilms. Reporte de caso
}

\author{
Wilms tumor. Case report \\ Vicuña Pozo María Fernanda', Arboleda Bustán Jenny Elizabeth', \\ García Andrade Jorge Alejandro ${ }^{1}$
}

VOLUMEN 39 | No 1 | SEPTIEMBRE 2021

FECHA DE RECEPCIÓN: $\quad$ 05/06/2020 FECHA DE APROBACIÓN: 02/06/2021 FECHA PUBLICACIÓN: 16/09/2021

1. Hospital Pediátrico Baca Ortiz. Urología. Quito - Ecuador

\begin{tabular}{r|l} 
Caso & Clinical \\
Clínico & Case
\end{tabular}

DOI: https://doi.org/10.18537/RFCM.39.01.07

Correspondencia:

fernandapozo1984ahotmail.com

Dirección:

Magdalena Adoum Juana Terrazas 60-1

Código Postal:

170144

Celular:

0998716922

Quito - Ecuador

\section{RESUMEN}

Introducción: el Tumor de Wilms es una neoplasia maligna embrionaria, representa el $6 \%$ de todos los cánceres pediátricos, cuyo origen ha sido atribuido a trastornos en la histogénesis renal, al parecer se desarrolla cuando los tejidos blastemales metanéfricos no maduran adecuadamente.

Caso clínico: paciente masculino de 3 años con un cuadro de pérdida de peso, estreñimiento e hipertensión arterial. Al examen físico de abdomen se palpa masa a nivel de hipocondrio y flanco izquierdo; en ecografía renal se observa riñón izquierdo que en su polo superior se encuentra reemplazado por voluminosa masa sólida con calcificaciones vascularizada. La tomografía de abdomen confirma sospecha diagnóstica, evidenciando aumento de volumen del riñón izquierdo y que se encuentra desplazado caudalmente por una masa isodensa que parte del polo superior. Se procede a realizar nefrectomía izquierda más vaciamiento ganglionar y se confirma el diagnóstico de Tumor de Wilms con el estudio histopatológico. El paciente ha permanecido asintomático asistiendo a controles por consulta externa una vez al año.

Conclusión: caso clínico de niño con masa abdominal diagnosticado y tratado de manera oportuna en un hospital de tercer nivel.

Palabras clave: tumor de wilms, nefrectomía, hospitales pediátricos, diagnóstico por imagen. 


\section{ABSTRACT}

Introduction: Wilms tumor is an embryonic malignant neoplasm, it represents $6 \%$ of all pediatric cancers, its origin has been attributed to disorders in renal histogenesis, apparently it develops when metanephric blastemal tissues do not mature properly.

Clinical case: It is a case of a 3-years-old male patient with weight loss, constipation and high blood pressure. On physical examination of the abdomen, a mass was palpated at the level of the hypochondrium and left flank; the renal ultrasound shows the left kidney that in its upper pole is replaced by a voluminous solid mass with vascularized calcifications. The abdominal tomography confirms the diagnosis, showing an increase in the volume of the left kidney and that is displaced caudally by an isodense mass that starts from the upper pole. A left nephrectomy plus lymph node dissection was performed and the diagnosis of Wilms tumor was confirmed with the histopathological study. The patient has remained asymptomatic, attending outpatient check-ups once a year.

Conclusion: clinical case of a child with an abdominal mass diagnosed and treated in a timely manner in a third level hospital.

Key words: wilms tumor, nephrectomy, hospitals pediatric, diagnostic imaging.

\section{INTRODUCCIÓN}

El Tumor de Wilms o nefroblastoma, neoplasia maligna del riñón, embrioma renal es el más frecuente en la edad pediátrica, representa el 6\% de todos los casos pediátricos ${ }^{1}$. Este se compone de células embrionarias blásticas estromales y epiteliales. El diagnóstico se realiza por ecografía, TAC abdominal o RNM. El diagnóstico es clínico e imagenológico, pero el definitivo es histopatológico². El tratamiento puede consistir en resección quirúrgica, quimioterapia y radioterapia. La supervivencia global es alrededor del $73 \%{ }^{3}$. Es muy importante el manejo de masas abdominales ya que, con el transcurso de los años, los avances en el diagnóstico y tratamiento del Tumor de Wilms mejoraron en gran medida las perspectivas (pronóstico) para los niños que sufren esta enfermedad.

\section{PRESENTACIÓN DEL CASO}

Niño de 3 años de edad que acude a consulta por pérdida de peso, acompañado de estreñimiento sin causa aparente de 4 meses de evolución, motivo por el que es derivado al hospital de segundo nivel de atención donde es valorado; al examen físico presenta hipertensión arterial, se palpa masa abdominal, se realiza ecografía que evidencia compromiso en riñón izquierdo, instaurándose tratamiento antihipertensivo y es referido al Hospital Pediátrico Baca Ortiz, la historia clínica no describe antecedentes patológicos personales ni familiares de importancia, con inmunizaciones completas para la edad de acuerdo al MSP, sin antecedentes patológicos quirúrgicos ni alergias, el nivel socio económico es medio, con alimentación adecuada para su edad. Al ingreso, paciente irritable al manejo activo, hidratado y afebril, presenta tensión arterial de 135/80 $\mathrm{mmHg}$, frecuencia cardíaca $100 \mathrm{Ipm}$, peso 13.3 kilogramos. Abdomen suave depresible se palpa masa a nivel de hipocondrio y flanco izquierdo que se extiende hasta la línea media, no móvil, no dolorosa al tacto y de bordes irregulares. Genitales masculinos normales. Por lo descrito, se decide ingreso y se realizan exámenes complementarios.

Exámenes de laboratorio: neutropenia, linfocitopenia, elemental y microscópico de orina: sangre 1 por campo hematíes 2 por campo, gram negativo. 


\section{Tabla $N^{\circ} 1$}

Química sanguínea

\begin{tabular}{lcc}
\hline Examen & Valor & Rango referencia \\
\hline Fosfatasa alcalina & $77.20 \mathrm{IU} / \mathrm{l}$ & $142.00-335.00$ \\
Deshidrogenasa láctica & $708.90 \mathrm{IU} / \mathrm{L}$ & $0.00-615.00$ \\
Aspartato aminotransferasa & $35.80 \mathrm{U} / \mathrm{L}$ & $0.00-56.00$ \\
Alanina aminotransferasa & $14.30 \mathrm{U} / \mathrm{L}$ & $0.00-39.00$ \\
\hline
\end{tabular}

Exámenes de imagen: ecografía de abdomen, riñón izquierdo el polo superior se encuentra reemplazado porvoluminosa masa de ecoestructura heterogénea sólida con calcificaciones vascularizada al estudio doppler, desplaza a las estructuras adyacentes en sentido caudal y derecho, mide $9.2 \times 9.6 \times 7.2 \mathrm{~cm}$, por el tamaño no es posible valorar vasos renales (Imagen $N^{\circ} 1$ ).

Tomografía de abdomen: aumento de volumen riñón izquierdo que es desplazado caudalmente por una masa isodensa que parte del polo superior, mide $12 \times 9.5 \mathrm{~cm}$ (Imagen $\mathrm{N}^{\circ} 2$ ). TAC de tórax no evidencia lesiones ocupantes. Se interconsulta al servicio de oncología quienes recomiendan cirugía.
Se realiza nefrectomía izquierda más vaciamiento ganglionar, en el tras quirúrgico se encuentra masa retroperitoneal dependiente de riñón izquierdo de gran tamaño, ganglios para-aórticos comprometidos. (Imagen $\mathrm{N}^{\circ} 3$ ). Al informe de patología el peso de la muestra 729 gramos, tamaño del tumor $12 \times 10 \times 9.2 \mathrm{~cm}$, fascia de gerota ausente, seno renal compromiso de los espacios linfovasculares, cápsula renal no identifica extensión más allá de ella. Sistema de estadiaje para tumores aparte de carcinoma de células renales del Children's Oncology Group: estadio local II. Hallazgos adicionales ganglios linfáticos con vasos dilatados (Imagen $\mathrm{N}^{\circ} 4$ ).

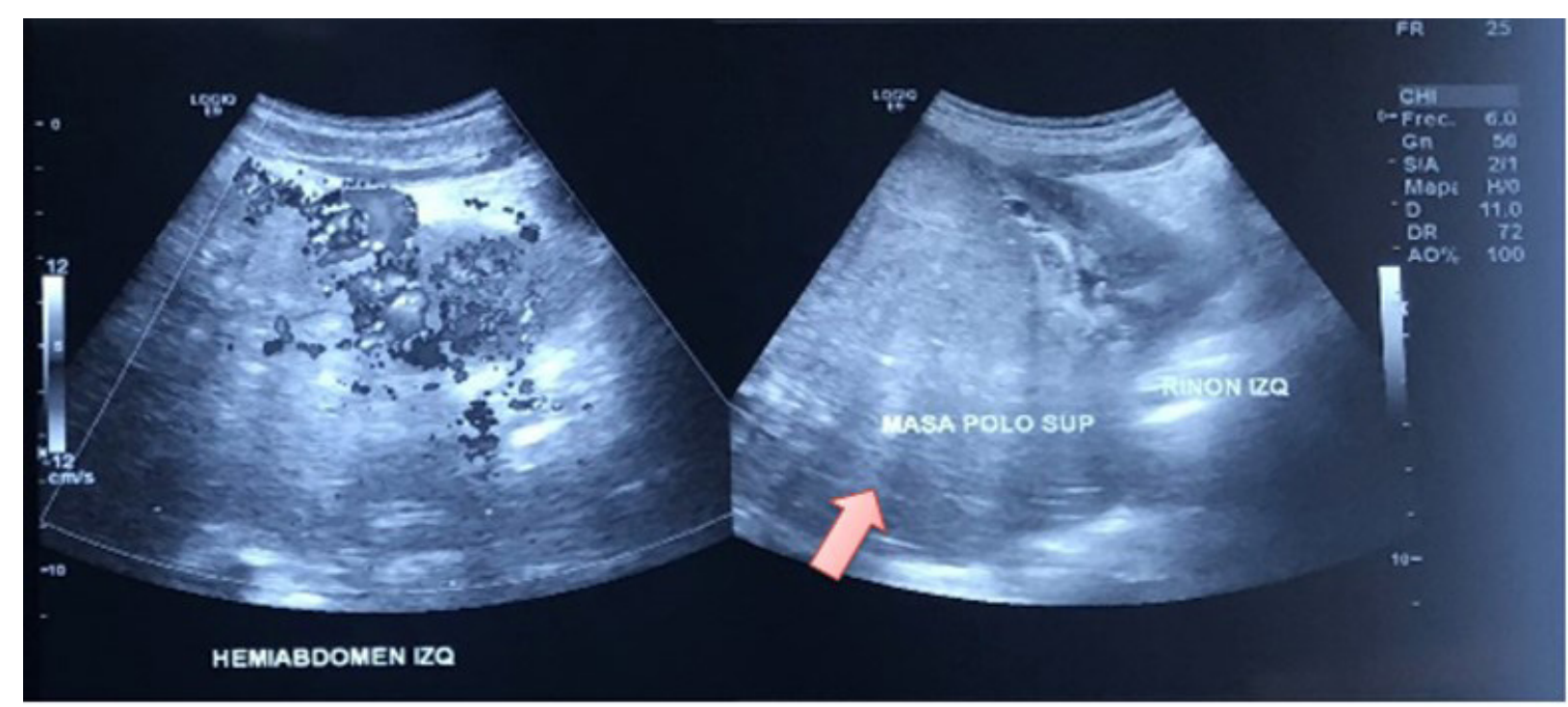

Imagen $N^{\circ} 1$

Ecografía renal: el polo superior se encuentra reemplazado por voluminosa masa de ecoestructura heterogénea sólida. 


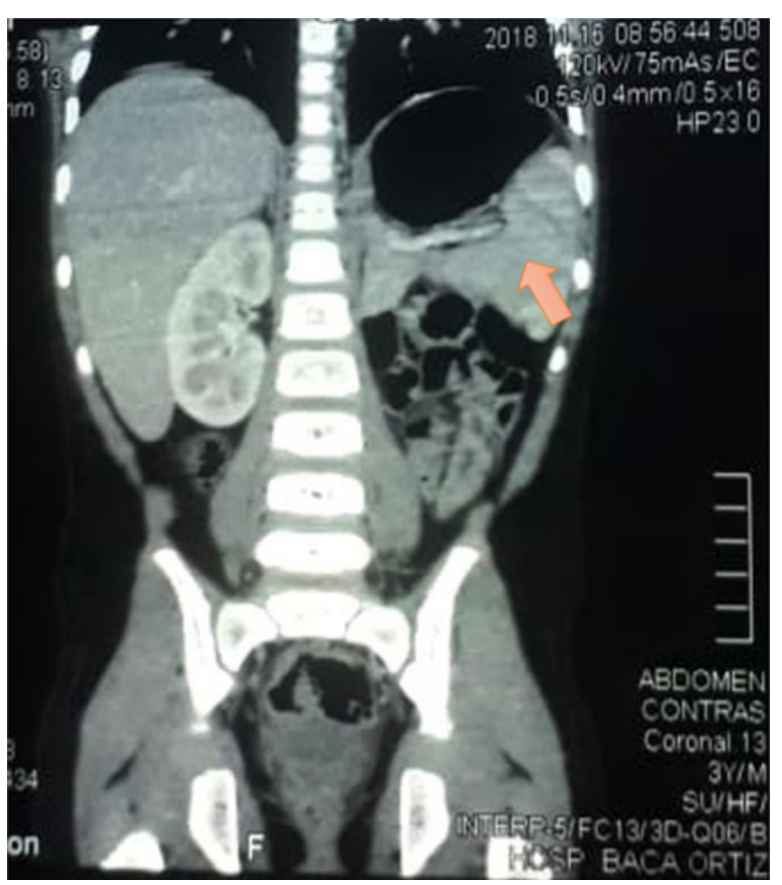

Imagen $\mathrm{N}^{\circ} 2$

Tomografía axial computarizada de abdomen: aumento de volumen riñón izquierdo y ubicado caudalmente por una masa isodensa.

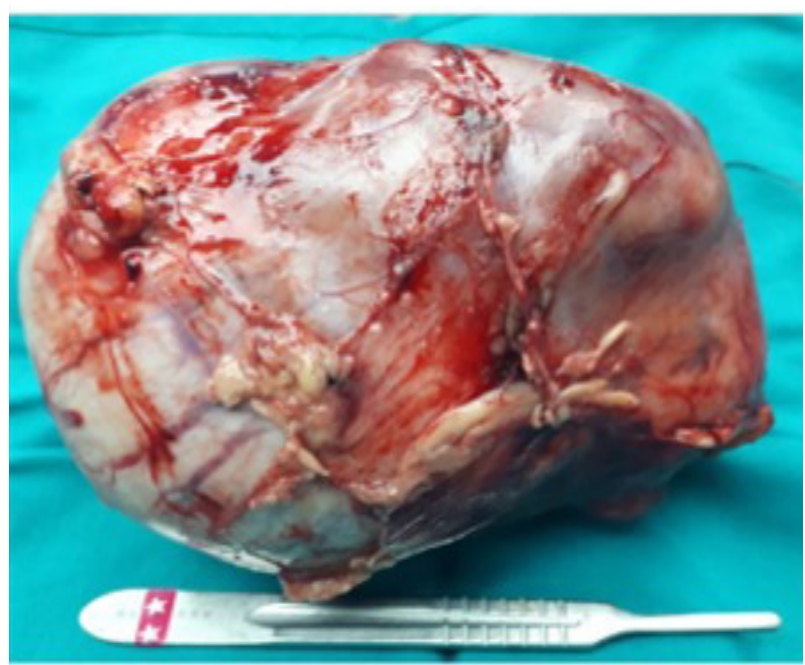

Imagen $\mathrm{N}^{\circ} 3$

Visión operatoria de tumor renal, mide $12 \times 10 \times 9.2$ centímetros con un peso 729 gramos.

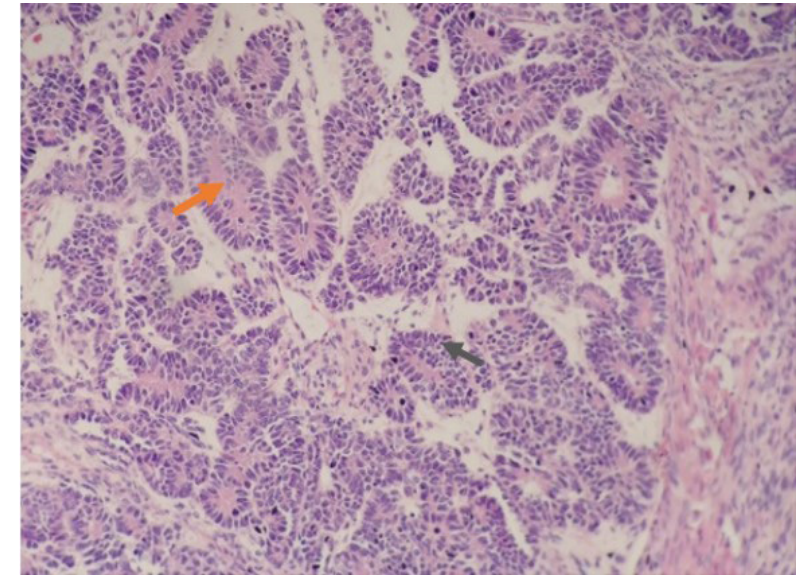

\section{Imagen $\mathrm{N}^{\circ} 4$}

Visión del histopatológico, lesiones malignas trifásicas con componentes blastemales, muestra patrón difuso de células pequeñas y azules

La evolución postoperatoria del paciente fue favorable, estuvo ingresado por 8 días, no hubo sangrado, no se evidenciaron complicaciones postquirúrgicas tempranas, sonda vesical permaneció por 5 días, no se dejó drenaje, tolerancia oral adecuada y se mantuvo el tratamiento antihipertensivo, por lo que se dio el alta a los 8 días y fue manejado por consulta externa de oncología según el criterio de la Nacional Wilms Tumor Study Group (NWTSG).

\section{DISCUSIÓN}

El Tumor de Wilms o nefroblastoma es el segundo cáncer intraabdominal más común de la infancia y la quinta neoplasia maligna más frecuente en la edad pediátrica. Representa aproximadamente el $6 \%$ de todos los cánceres pediátricos y es el tumor renal más frecuente (más del $95 \%$ del total de tumores renales pediátricos) $)^{4}$. Se produce entre los 2 a 5 años de edad, siendo el $95 \%$ de los niños diagnosticados antes de los 10 años $^{5}$, la edad de nuestro paciente es de 3 años.

Estudios revelaron la edad menor de 2 años como un factor pronóstico favorable, mientras que la edad mayor a 4 años se ha descrito como un factor pronóstico adverso ${ }^{6}$, el paciente está en una edad intermedia y hasta el momento se ha mantenido asintomático. Está formado por células de la estirpe de los tres tipos celulares embrionarios: blastema, sarcomatoso y epitelial. Su origen es genético y se asocia a la selección del brazo corto 
del cromosoma 11, en donde se encuentra el gen supresor de tumores WT1.

El signo más frecuente es el hallazgo incidental de una masa abdominal, que es descubierto por los padres, la masa suele ser lisa y firme bien demarcado, seguido de hematuria, pero alrededor del $25 \%$ de los casos presenta hematuria microscópica, en algunos casos puede asociarse a hipertensión arterial por isquemia renal secundaria a la presión ejercida sobre la arteria renal. En signos menos específicos puede incluir anorexia, pérdida de peso, fiebre, anemia y malestar general ${ }^{7}$, en el paciente se encontró masa abdominal e hipertensión arterial por lo que se relaciona con los signos más frecuentes en este tipo de tumor.

El diagnóstico inicial es clínico e imagenológico; sin embargo, el definitivo ante un tumor de Wilms es histopatológico ${ }^{8}$; en nuestro paciente se confirmó el resultado con este estudio. Para la aproximación diagnóstica, el ultrasonido doppler, la TAC y RNM son útiles tanto para definir la presencia y características del tumor $^{9}$, como también para delimitar la presencia, extensión y localización de los sitios de metástasis a distancia; el paciente tuvo la ecografía y tomografía pulmonar y abdominal que descarta metástasis a distancia.

Existen varios protocolos de tratamiento según la National Wilms Tumor StudyGroup ${ }^{10}$, y la Estatificación de la Sociedad Internacional de Oncología Pediátrica (SIOP) de los tumores renales de la infancia $(2001)^{11}$ el mismo que fue utilizado y lo divide en estadios. Nuestro paciente se caracteriza por cumplir con el estadio II que el tumor se extiende fuera del riñón, pero es totalmente resecado: la cápsula, los tejidos adyacentes, vasos del seno renal y vena cava pueden estar implicados.

Existen dos criterios según el NWTSG que defiende la nefrectomía inmediata para asegurar el diagnóstico histológico y la estatificación precisa, mientras que la SIOP en Europa defiende la quimioterapia preoperatoria para promover la reducción del tumor y de este modo facilitar la cirugía, evitando rupturas de la cápsula tumoral, realizando estatificación tras la misma ${ }^{12}$.

Por lo tanto, en SIOP, la quimioterapia postoperatoria para tumor de Wilms se intensificó con el objetivo de mejorar la supervivencia ${ }^{13}$, el paciente según el estadio II se realizó nefrectomía radical después de lo cual recibió tratamiento con quimioterapia por 8 ciclos.

\section{CONCLUSIÓN}

Los niños con Tumor de Wilms pueden cursar con una masa abdominal asintomática, algunos casos son detectados de casualidad por los padres, cuidadores o pediatras; en el paciente el hallazgo de la masa abdominal resultó incidental, por lo que es necesario siempre la valoración médica periódica para un diagnóstico oportuno.

\section{ASPECTOS BIOÉTICOS}

El presente trabajo se realizó bajo el consentimiento informado de los padres del paciente, con total confidencialidad de datos personales.

\section{INFORMACIÓN DE LOS AUTORES}

- Vicuña Pozo María Fernanda. Médico General. Hospital Pediátrico Baca Ortiz. Urología. QuitoPichincha-Ecuador.

e-mail: fernandapozo1984@hotmail.com

ORCID: https://orcid.org/0000-0003-2985-634X

- Arboleda Bustán Jenny Elizabeth. Médica General. Especialista en Cirugía Pediátrica. Master en Urología Pediátrica. Hospital Pediátrico Baca Ortiz. Urología. Quito-Pichincha-Ecuador.

e-mail: elizabethab2011@gmail.com

ORCID: https://orcid.org/0000-0002-6605-5173

- García Andrade Jorge Alejandro. Doctor en Medicina y Cirugía. Especialidad de Cirugía Infantil. Hospital Pediátrico Baca Ortiz. Urología. Quito-Pichincha-Ecuador.

e-mail: jorge.garcia@hbo.gob.ec

ORCID: https://orcid.org/0000-0001-7690-547X

\section{CONTRIBUCIÓN DE LOS AUTORES}

VPMF: concepción y diseño del trabajo con el correspondiente análisis e interpretación de los datos. Capacidad de responder de todos los aspectos del artículo.

ABJE: redacción y revisión crítica del manuscrito. Aprobación de la versión final. Capacidad de responder de todos los aspectos del artículo. 
GAJA: redacción y revisión crítica del manuscrito. Aprobación de la versión final. Capacidad de responder de todos los aspectos del artículo.

\section{CONFLICTO DE INTERESES}

Los autores no reportan conflictos de intereses.

\section{FUENTES DE FINANCIAMIENTO}

\section{Autofinanciado}

\section{REFERENCIAS BIBLIOGRÁFICAS}

1. Green DM. Considerations in the Diagnosis and Management of Pediatric Patients with Favorable Histology Wilms Tumor Who Present with Only Pulmonary Nodules. Pediatr Blood Cancer. 2016 Apr; 63(4):58992. Doi: $10.1002 / p b c .25840$

2. Illade L, Hernandez-Marques C, Cormenzana M, Lassaletta Á, Andión Catalán M, Ruano $D$, et al. Tumor de Wilms: revisión de nuestra experiencia en los últimos 15 años. An Pediatr (Barc). 2018;88(3):140-9. Doi: 10.1016/j.anpedi.2017.03.019.

3. Han Q, Li K, Dong K, Xiao X, Yao W, Liu G. Clinical features, treatment, and outcomes of bilateral Wilms' tumor: A systematic review and meta-analysis. J Pediatr Surg. 2018;53(12):2465-9. Doi: 10.1016/j. jpedsurg.2018.08.022. Epub 2018 Sep 1. PMID: 30274708.

4. Chu DI, Ehlayel AM, Ginsberg JP, Meyers KE, Benton M, Thomas M, et al. Kidney outcomes and hypertension in survivors of Wilms tumor: A prospective cohort study. $J$ Pediatr. 2021; 230:215-220.e1. Doi: 10.1016/j.jpeds.2020.12.005

5. Vanden Berg RNW, Bierman EN, Van Noord M, Rice HE, Routh JC. Nephron-sparing surgery for Wilms tumor: A systematic review. Urol Oncol. 2016;34(1):24-32. Doi: 10.1016/j. urolonc.2015.07.00

6. Herrera-ToroN, Peña-AguirreL, ArangoRaveME. Tumor de Wilms: experiencia de 12 años en dos hospitales de alto nivel en Medellín, Colombia. Iatreia [Internet].
1 de abril de 2019 [citado 11 de marzo de 2021];32(2):82-1. Doi: https://doi. org/10.17533/udea.iatreia.13

7. Laboni DSM, Chi Y-Y, Kim Y, Dome JS, Fernandez CV. Outcome of Wilms tumor patients with bone metastasis enrolled on National Wilms Tumor Studies 1-5: A report from the Children's Oncology Group. Pediatr Blood Cancer. 2019;66(1): e27430. Doi: 10.1002/pbc.27430. Epub 2018 Aug 30. PMID: $30160355 ;$ PMCID: PMC6249096.

8. Hohenstein P, Pritchard-Jones K, Charlton J. The yin and yang of kidney development and Wilms' tumors. Genes Dev. 2015;29(5):46782. Doi: 10.1101/gad.256396.114. PMID: 25737276 ; PMCID: PMC4358399.

9. Dumba $\mathrm{M}$, Jawad $\mathrm{N}$, McHugh $\mathrm{K}$. Neuroblastoma and nephroblastoma: a radiological review. Cancer Imaging. 2015;15(1):5. URL: http://dx.doi.org/10.1186/ s40644-015-0036-2

10. K.F. Wong, R.C. Reulen, D.L. Winter, J. Guha, M.M. Fidler, J. Kelly, et al. Risk of adverse health and social outcomes up to 50 years after Wilms tumor: The British Childhood Cancer Survivor Study. J Clin Oncol. 2016; 34: 1772-1779. URL: http:// dx.doi.org/10.1200/JCO.2015.64.4344

11. Van den Heuvel-Eibrink MM, van Tinteren $\mathrm{H}$, Bergeron C, Coulomb-L'Hermine A, de Camargo B, Leuschner I, et al. Outcome of localised blastemal-type Wilms tumour patients treated according to intensified treatment in the SIOP WT 2001 protocol, a report of the SIOP Renal Tumour Study Group (SIOP-RTSG). Eur J Cancer. 2015;51(4):498506. Doi: 10.1016/j.ejca.2014.12.011. Epub 2015 Jan 12. PMID: 25592561.

12. Gooskens SL, Segers H, Pritchard-Jones K, Graf N, Dome JS, van den Heuvel-Eibrink MM. Wilms Tumor. van den Heuvel-Eibrink MM, Princess Maxima Center for Pediatric Oncology/Hematology, Lundlaan 6, 3584 EA Utrecht, The Netherlands, editors. Codon Publications; 2016. Doi: 10.15586/codon. wt.2016.ch2 
13. Rabeh W, Akel S, Eid T, Muwakkit S, Abboud $\mathrm{M}$, El Solh $\mathrm{H}$, et al. Wilms tumor: Successes and challenges in management outside of cooperative clinical trials. Hematol Oncol Stem Cell Ther. 2016;9(1):20-5. Doi: 10.1016/j.hemonc.2015.12.006. Epub 2016 Jan 18. PMID: 26802622. 